\title{
Quantum Description for the Decay of NSNS Brane-Antibrane Systems
}

\author{
Hongsu Kim ${ }^{1}$ \\ Astronomy Program, SEES, Seoul National University \\ Seoul, 151-742, KOREA
}

\begin{abstract}
The stringy description for the instabilities in the $R R$ charged $D_{p}-\bar{D}_{p}$ pairs is now well understood in terms of the open string tachyon condensation. The quantum interpretation presumably via the stringy description for the instabilities in the NSNScharged $F 1-\bar{F} 1$ and $N S 5-\overline{N S} 5$ pairs in IIA/IIB theories, however, has not been fully established yet. This would be partly because of the absence (for the $F 1$ $\bar{F} 1$ case) or our relatively poor understanding (for the $N S 5-\overline{N S} 5$ case) of their worldvolume (gauge theory) dynamics. In the present work, using the well-known quantum description for instabilities in the $R R$-charged $D_{p}-\bar{D}_{p}$ systems and in the Mtheory brane-antibrane systems and invoking appropriate string dualities, the stringy nature of the instabilities in the $N S N S$-charged $F 1-\bar{F} 1$ and $N S 5-\overline{N S} 5$ systems both at strong and at weak couplings has been uncovered. For the annihilations to string vacua, the quantum, stringy interpretations are simple extensions of Sen's conjecture for those in $R R$-charged brane-antibrane systems.
\end{abstract}

PACS numbers : 11.25.Sq, 04.65.+e, 11.10.Kk

\footnotetext{
${ }^{1}$ hongsu@astro.snu.ac.kr
} 


\section{Introduction}

The stringy description for the instabilities in the $R R$ charged $D_{p}-\bar{D}_{p}$ pairs [1] is now well understood in terms of the open string tachyon condensation. Consider a system consisting of a certain number $N$ of coincident $D_{P}$-branes separated by some distance from a system of $N$ coincident $\bar{D}_{p}$-branes, for simplicity, in flat $R^{10}$. This system differs from the BPS system of $2 N D_{p}$-branes by the orientation reversal on the antibranes. In this system, the branes and the antibranes each break a different half of the original supersymmetry and the whole configuration is non-supersymmetric or non-BPS and hence is unstable. As a result, there is a combined gravitational and $(\mathrm{RR})$ gauge attractive force between the branes and the antibranes at some large but finite separation leading to the semi-classical instability. At the separation of order the string scale $\sim \sqrt{\alpha^{\prime}}=l_{s}$, in particular, the open string connecting a $D_{p}$-brane to a $\bar{D}_{p}$-brane becomes tachyonic. What then would be the eventual fate or endpoint of this unstable $D_{p}-\bar{D}_{p}$-system ? According to Sen [2], the endpoint could be the supersymmetric vacuum via the open string tachyon condensation. Later on in section 2.2 , we shall be more specific on this. The quantum interpretation, again presumably via the stringy description for the instabilities in the $N S N S$-charged $F 1-\bar{F} 1$ and $N S 5-N \bar{S} 5$ pairs in IIA/IIB theories, however, has not been fully established yet [3]. This would be partly due to the absence (for the $F 1-\bar{F} 1$ case) or our relatively poor understanding (for the NS5 - NS5 case) of their worldvolume (gauge theory) dynamics. (Later on in the concluding remarks, we shall summarize particularly some of the collected wisdom on the nature of NS5-brane worldvolume dynamics uncovered thus far.) And certainly, this nature is reflected in the intersection rules saying that fundamental strings do not end on themselves nor on $N S 5$-branes, i.e., neither $(0 \mid F 1, F 1)$ nor $(0 \mid F 1, N S 5)$ exists. As a result, unlike the $R R$-charged $D_{p}$-brane case in which the fundamental strings ending on $D_{p}$ or $\bar{D}_{p}$ essentially provides the worldvolume dynamics of the branes and generates the instabilties in the $D_{p}-\bar{D}_{P}$ systems, in this $N S N S$-charged $F 1-\bar{F} 1$ and $N S 5-N \bar{S} 5$ systems, the quantum, elementary entity both to provide the worldvolume dynamics and to generate the instabilities in the brane-antibrane systems is missing [3]. This is certainly an embarrassing state of affair if we realize the fact that these $N S N S$-charged brane-antibrane pairs are just $U$-duals of $R R$-charged ones for which the quantum, stringy description for the instabilities is well-established in terms of Sen's conjecture of open string tachyon condensation [2]. In the present work, therefore, using the well-known quantum description for instabilities in the $R R$-charged $D_{p}-\bar{D}_{p}$ systems and in the M-theory brane-antibrane systems and invoking 
appropriate string dualities, the stringy nature of the instabilities in the $N S N S$-charged $F 1-\bar{F} 1$ and $N S 5-N \bar{S} 5$ systems both at strong and at weak couplings has been uncovered. And particularly for the annihilations to string vacua, the quantum, stringy interpretations are simple extensions of Sen's conjecture given for those in $R R$-charged brane-antibrane systems.

\section{Instabilities in IIB theory $(F 1-\bar{F} 1, N S 5-N S 5)$ sys- tems}

\subsection{Supergravity description of the instability}

In this section, we would like to demonstrate in an explicit manner that via the $S$-duality, one can actually obtain the supergravity solutions representing $F 1-\bar{F} 1$ and $N S 5-N \bar{S} 5$ systems from those representing $D 1-\bar{D} 1$ and $D 5-\bar{D} 5$ systems rspectively. Thus to this end, we begin with the type IIB supergravity action in string frame

$$
\begin{aligned}
S_{I I B} & =\frac{1}{2 \kappa^{2}} \int d^{10} x \sqrt{g}\left\{e^{-2 \phi}\left[R+4(\nabla \phi)^{2}-\frac{1}{12} H_{[3]}^{2}\right]-\frac{1}{2}(\partial \chi)^{2}\right. \\
& \left.-\frac{1}{12} F_{[3]}^{2}-\frac{1}{240} F_{[5]}^{2}\right\}+\frac{1}{4 \kappa^{2}} \int A_{[4]} \wedge d A_{[2]} \wedge H_{[3]}
\end{aligned}
$$

where $\kappa^{2}=8 \pi G, H_{[3]}=d B_{[2]}$ is the field strength of the $N S N S$ tensor field $B_{[2]}$ and $F_{[3]}=d A_{[2]}-\chi H_{[3]}, F_{[5]}=d A_{[4]}+A_{[2]} \wedge H_{[3]}$ are the $R R$ field strengths. And when one writes the type IIB supergravity action as above, it is implicit that one never asks that the self-(Hodge) duality condition on the 5 -form $R R$ field strength $F_{[5]}$ follows from the variation of this action but instead is assumed to be imposed afterwards by hand, $* F_{[5]}=F_{[5]}$. Now note that the IIB theory equations of motion that result by extremizing the IIB theory action given above are invariant under a $S L(2, R)$ symmetry (and it is broken to $S L(2, Z)$ in the full type IIB string theory) under which the fields transform as follows. Namely define

$$
\lambda=\chi+i e^{-\phi}, \quad H=\left(\begin{array}{c}
B_{[2]} \\
A_{[2]}
\end{array}\right)
$$

where $\chi$ and $\phi$ are the $R R$ scalar (axion) and the $N S N S$ scalar (dilaton) respectively appeared in the IIB theory action given above. Then, under a $S L(2, R)$ transformation represented by the matrix

$$
U=\left(\begin{array}{ll}
a & b \\
c & d
\end{array}\right) \in S L(2, R) \quad \text { with } \quad a d-b c=1
$$


the scalars and the 2-form potentials transform according to

$$
\lambda \rightarrow \frac{a \lambda+b}{c \lambda+d}, \quad H \rightarrow U H=\left(\begin{array}{ll}
a & b \\
c & d
\end{array}\right) H
$$

with the other fields remaining invariant. Now, consider a particular case of this $S L(2, R)$ transformation in which one sets the $R R$ scalar (axion) to zero, $\chi=0$. This particular $S L(2, R)$ transformation amounts to choosing $(a=0, b=1, c=-1, d=0)$ under which the fields transform as

$$
\lambda^{\prime}=-\frac{1}{\lambda}\left(\text { or } e^{-\phi^{\prime}}=e^{\phi}\right), \quad\left(\begin{array}{c}
B_{[2]}^{\prime} \\
A_{[2]}^{\prime}
\end{array}\right)=\left(\begin{array}{c}
A_{[2]} \\
-B_{[2]}
\end{array}\right), \quad g_{\mu \nu}^{\prime}=e^{-\phi} g_{\mu \nu}
$$

(in the string frame) and is referred to as $S$-duality.

With this preparation, we first start with the supergravity solution representing the $D 1-\bar{D} 1$ pair of type IIB theory [6].

$$
\begin{aligned}
d s_{10}^{2} & =H^{-1 / 2}\left[-d t^{2}+d x_{1}^{2}\right]+H^{1 / 2}\left[\sum_{m=2}^{6} d x_{m}^{2}+\left(\Delta+a^{2} \sin ^{2} \theta\right)\left(\frac{d r^{2}}{\Delta}+d \theta^{2}\right)+\Delta \sin ^{2} \theta d \phi^{2}\right] \\
e^{2 \phi} & =H, \\
A_{[2]} & =\left[\frac{2 m a \cos \theta}{\Sigma}\right] d x_{1} \wedge d t, \quad F_{[3]}=d A_{[2]}
\end{aligned}
$$

where the "modified" harmonic function is given by $H(r)=\Sigma /\left(\Delta+a^{2} \sin ^{2} \theta\right)$ and $\Sigma=$ $r^{2}-a^{2} \cos ^{2} \theta, \Delta=r^{2}-2 m r-a^{2}$ with $m$ being the ADM mass of each $D$-brane. Then the ADM mass of the whole $D 1-\bar{D} 1$ system is $M_{A D M}=2 m$ which should be obvious as it would be the sum of ADM mass of each brane when they are well separated. The parameter $a$ can be thought of as representing the proper distance between the brane and the antibrane [3. Now, applying the $S$-duality transformation laws given above to this $D 1-\bar{D} 1$ solution, one gets the following supergravity solution

$$
\begin{aligned}
d s_{10}^{2} & =H^{-1}\left[-d t^{2}+d x_{1}^{2}\right]+\sum_{m=2}^{6} d x_{m}^{2}+\left(\Delta+a^{2} \sin ^{2} \theta\right)\left(\frac{d r^{2}}{\Delta}+d \theta^{2}\right)+\Delta \sin ^{2} \theta d \phi^{2}, \\
e^{2 \phi} & =H^{-1} \\
B_{[2]} & =\left[\frac{2 m a \cos \theta}{\Sigma}\right] d x_{1} \wedge d t, \quad H_{[3]}=d B_{[2]}
\end{aligned}
$$

which is indeed the $F 1-\bar{F} 1$ solution of IIA/IIB theory $[6]$.

Next, we start with the supergravity solution representing the $D 5-\bar{D} 5$ pair of IIB theory 6 .

$$
d s_{10}^{2}=H^{-1 / 2}\left[-d t^{2}+\sum_{i=1}^{5} d x_{i}^{2}\right]+H^{1 / 2}\left[d x_{6}^{2}+\left(\Delta+a^{2} \sin ^{2} \theta\right)\left(\frac{d r^{2}}{\Delta}+d \theta^{2}\right)+\Delta \sin ^{2} \theta d \phi^{2}\right],
$$




$$
\begin{aligned}
e^{2 \phi} & =H^{-1} \\
A_{[2]}^{m} & =\left[\frac{2 m r a \sin ^{2} \theta}{\Delta+a^{2} \sin ^{2} \theta}\right] d x_{6} \wedge d \phi, \quad F_{[3]}^{m}=d A_{[2]}^{m} .
\end{aligned}
$$

Similarly, by applying the $S$-duality transformation law to this $D 5-\bar{D} 5$ solution, one gets the following supergravity solution

$$
\begin{aligned}
d s_{10}^{2} & =-d t^{2}+\sum_{i=1}^{5} d x_{i}^{2}+H\left[d x_{6}^{2}+\left(\Delta+a^{2} \sin ^{2} \theta\right)\left(\frac{d r^{2}}{\Delta}+d \theta^{2}\right)+\Delta \sin ^{2} \theta d \phi^{2}\right] \\
e^{2 \phi} & =H, \\
B_{[2]}^{m} & =\left[\frac{2 m r a \sin ^{2} \theta}{\Delta+a^{2} \sin ^{2} \theta}\right] d x_{6} \wedge d \phi, \quad H_{[3]}^{m}=d B_{[2]}^{m}
\end{aligned}
$$

which indeed can be identified with the $N S 5-N \bar{S} 5$ solution of IIA/IIB theory [6]. That these IIB theory $D 1-\bar{D} 1, D 5-\bar{D} 5$ pairs and their $S$-dual $F 1-\bar{F} 1, N S 5-N \bar{S} 5$ pairs represented by the supergravity solutions given above indeed exhibit semi-classical instabilities in terms of the appearance of the conical singularities can be found in detail in our earlier work [3] in which $D 6-\bar{D} 6$ system has been taken for explicit demonstration. Indeed, the nature of semi-classical instabilities owned by these $R R / N S N S$-charged brane-antibrane systems in terms of the conical singularities is very reminiscent of essentially the same conical singularity structure in the Bonnor's magnetic dipole solution [7] in Einstein-Maxwell theory. We now attempt to describe briefly the nature of these semi-classical instabilities. consider the symmetry axis $\theta=0, \pi$ connecting the brane and the antibrane. Then one can see that the conical singularities arise both along the semi-infinite axes extending from the (anti)brane to infinity and along the line segment between the brane and the antibrane. One, however, can immediately realise that both of the two conical singularities cannot be eliminated at the same time. Then the usual option one takes is to remove the conical singularity along the line segment between the two at the expense of the appearance of the conical angle deficits along the semi-infinite axes. This, in turn, implies the presence of the cosmic strings providing tension that pulls the brane and the antibrane apart against the collapse due to the combined gravitational and gauge attractions. Nevertheless, the semi-classical instabilities owned by these brane-antibrane systems still manifest themselves since it is not hard to realise that the cosmic strings can only suspend the brane-antibrane systems in an unstable equilibrium configuration. Namely, it can be demonstrated that this unstable equilibrium is indeed vulnerable since if one brings the brane and the antibrane close to each other, they always collide and merge completely. To see this, note first that the parameter $a$ appearing in the supergravity solutions above can be regarded as representing the proper separation 
between the brane and the antibrane [3].

Now consider the $D 1-\bar{D} 1$ solution given in eq.(6). In the limit $a \rightarrow 0$, it becomes

$$
\begin{aligned}
d s_{10}^{2} & =\left(1-\frac{2 m}{r}\right)^{1 / 2}\left[-d t^{2}+d x_{1}^{2}\right] \\
& +\left(1-\frac{2 m}{r}\right)^{-1 / 2}\left[\sum_{m=2}^{6} d x_{m}^{2}+d r^{2}+r^{2}\left(1-\frac{2 m}{r}\right)\left(d \theta^{2}+\sin ^{2} \theta d \phi^{2}\right)\right] \\
e^{2 \phi} & =\left(1-\frac{2 m}{r}\right)^{-1}, \quad A_{[2]}=0
\end{aligned}
$$

where we used $\Sigma \rightarrow r^{2}, \Delta \rightarrow r^{2}(1-2 m / r)$, and hence $H \rightarrow(1-2 m / r)^{-1}$ as $a \rightarrow 0$. In this limit, the opposite $R R$ charges carried by $D 1$ and $\bar{D} 1$ annihilated each other since $A_{[2]}=0$ and the solution now has the topology of $R \times R^{7} \times S^{2}$. Particularly, the $S O(3)$-isometry in the transverse space implies that, as they approach, $D 1$ and $\bar{D} 1$ actually merge and as a result a curvature singularity develops at the center $r=0$.

Next we consider the $F 1-\bar{F} 1$ system given above in eq.(7) and again take the limit $a \rightarrow 0$.

$$
\begin{aligned}
d s_{10}^{2} & =\left(1-\frac{2 m}{r}\right)\left[-d t^{2}+d x_{1}^{2}\right]+\sum_{m=2}^{6} d x_{m}^{2}+d r^{2}+r^{2}\left(1-\frac{2 m}{r}\right)\left(d \theta^{2}+\sin ^{2} \theta d \phi^{2}\right) \\
e^{2 \phi} & =\left(1-\frac{2 m}{r}\right), \quad B_{[2]}=0 .
\end{aligned}
$$

In this limit, again it appears that the opposite electric $N S N S$ charges carried by $F 1$ and $\bar{F} 1$ annihilate each other since $B_{[2]}=0$ and the metric solution has the topology of $R \times R^{7} \times S^{2}$. Once again, the manifest $S O(3)$-isometry in the transverse space implies that, as they are brought together, $F 1$ and $\bar{F} 1$ actually collide and as a result a curvature singularity develops at the center $r=0$.

To summarize, as one can see in this supergravity descriptions, both $R R$ and $N S N S$-charged brane-antibrane systems are on equal footing in that they exhibit essentially the same semiclassical instabilities. And as the inter-brane distance gets smaller and smaller, say, towards the string scale $\sim \sqrt{\alpha^{\prime}}=l_{s}$, we expect that these semi-classical instabilities should be taken over by the associated quantum, stringy instabilities. As we mentioned in the introduction, however, actually we have an embarrassing state of affair since in the $N S N S$-charged case, the quantum entity that should take over the semi-classical instability at short length scales is missing. To be a little more concrete, we invoke Sen's conjecture [2] for the decay/annihilation of unstable $D$-branes via the open string tachyon condensation. From the stringy perspective based on the open string field theory, Sen suggested that the eventual fate of the non-BPS $D_{p}-\bar{D}_{p}$ system could be a supersymmetric vacuum via the open string 
tachyon condensation. Namely, the brane and the antibrane merge and annihilate each other completely since firstly, the opposite $R R$ charges are cancelled and secondly, the total energy of the system, upon merging, may vanish [2], $E_{t o t}=V\left(T_{0}\right)+2 M_{D}=0$. Thus according to this conjecture by Sen, the outcome of the brane-antibrane collision could be a complete annihilation into a supersymmetric vacuum. (We shall provide a detailed review of Sen's conjecture below in the next subsection.) In the "NS"-charged case, however, the situation changes. Namely, since fundamental string does not end on another F1 nor on NS5 (namely no intersection rules such as $(0 \mid F 1, F 1)$ or $(0 \mid F 1, N S 5)$ exists), there is, as a result, no stringy description available for the brane-antibrane annihilation in terms of open string tachyon condensation via Sen's mechanism. This absence of the quantum mechanism for the outcome of $F 1-\bar{F} 1$ or $N S 5-N \bar{S} 5$ annihilation is indeed a very unnatural state of affair in light of the fact that $F 1-\bar{F} 1$ and $N S 5-N \bar{S} 5$ systems are just $U$ duals to $D_{p}-\bar{D}_{p}$ systems as we have seen earlier. Certainly, therefore, a quantum, stringy description is in need for these $F 1-\bar{F} 1$ and $N S 5-\overline{N S} 5$ annihilations into (presumably) supersymmetric vacua and in the present work, we shall address this issue.

\subsection{Stringy description of the instability at strong coupling}

Evidently, there is a combined gravitational and $(R R)$ gauge attractions between the $R R$ charged $D_{p}$ and $\bar{D}_{p}(p=1,5)$ at some large but finite separation leading to the semiclassical instability. And it manifests itself in terms of the presence of conical singularities owned by the supergravity solutions representing $D 1-\bar{D} 1, D 5-\bar{D} 5$ pairs as mentioned above. As the inter-brane distance gets smaller and smaller toward, say, the distance of order $a \sim \sqrt{\alpha^{\prime}}=l_{s}$, this semi-classical description of the instability should be replaced by the quantum, stringy one that is represented by the tachyonic mode arising in the spectrum of open strings stretched between $D_{p}$ and $\bar{D}_{p}$. Then as our supergravity analysis given above indicates (in which the $N S N S$-charged $(F 1-\bar{F} 1, N S 5-\overline{N S} 5)$ systems are shown to be related, via the $S$-duality, to the $R R$-charged $(D 1-\bar{D} 1, D 5-\bar{D} 5)$ systems), in a similar manner, the quantum, stringy description of the instabilities in the $(F 1-\bar{F} 1, N S 5-N \bar{S} 5)$ systems should be related to that in the $(D 1-\bar{D} 1, D 5-\bar{D} 5)$ systems via the stringy (or brany) version of $S$-duality as well. This natural anticipation thus leads us to propose the following quantum interpretation of the instabilities that reside in the NSNS-charged braneantibrane systems. Thus we first start with the quantum description of the instabilities in the $(D 1-\bar{D} 1, D 5-\bar{D} 5)$ systems. Now consider the GSO projection for the open (super) string in which we use the convention that the $(-1)^{F}$ ( " $F$ " here denotes the worldsheet 
fermion number carried by the string state) eigenvalue of the $N S$ sector ground state is -1 . Then from the observation that for the NSNS sector, the closed string exchange interactions between the $D_{p}-D_{p}$ and between the $D_{p}-\bar{D}_{p}$ have the same sign whereas for the $R R$ sector, they have opposite sign, one can deduce, using the closed string channel-open string channel duality, that the GSO projection operator for strings in $D_{p}-D_{p}$ system is $(1 / 2)\left[1+(-1)^{F}\right]$, while that for strings in $D_{p}-\bar{D}_{p}$ system is $(1 / 2)\left[1-(-1)^{F}\right]$. Namely, the open strings stretched between $D_{p}$ and $\bar{D}_{p}$ have wrong GSO projection and thus develop tachyonic mode as the lowest-lying state. This troublesome result of having the tachyonic mode in the non-BPS $D_{p}-\bar{D}_{p}$ systems, however, has been circumvented by the idea of the unstable $D_{p}-\bar{D}_{p}$ system decay via the open string tachyon condensation. Particularly, according to the conjecture due to Sen [2], the condensation mechanism can be stated briefly as follows. Consider, for example, a coincident $D_{p}-\bar{D}_{p}$ pair in type IIB theory. Upon integrating out all the massive modes in the spectrum of open strings on the $D_{p}-\bar{D}_{p}$ worldvolume one should get the tachyon potential and it gets its maximum at the false vacuum expectation value (vev) $T=0$. Next, since there is a $U(1) \times U(1)$ Born-Infeld gauge theory living in the worldvolume of this $D_{p}-\bar{D}_{p}$ system, the tachyon field $T$ picks up a phase under each of these $U(1)$ gauge transformations. As a result, the tachyon potential $V(T)$ is a function only of $|T|$ and its minimum occurs at $T=T_{0} e^{i \theta}$ for some fixed true vev $T_{0}$. Then the essence of Sen's conjecture is the proposition that at $T=T_{0}$, the sum of the tension of $D$-brane and anti- $D$-brane and the (negative) minimum negative potential energy of the tachyon is exactly zero

$$
V\left(T_{0}\right)+2 M_{D}=0
$$

with $M_{D}=T_{D}$ being the $D$-brane tension. And the philosophy behind this conjecture is the physical insight that the endpoint of the brane-antibrane annihilation would be a string vacuum in which the supersymmetry is fully restored. Thus this is the standard way (at least at the moment) of describing the instabilities in the $R R$-charged brane-antibrane systems such as $(D 1-\bar{D} 1, D 5-\bar{D} 5)$ pairs in quantum, stringy terms.

Consider now, its $S$-dual picture. Clearly, the $S$-dual of the $D 1-\bar{D} 1$ system with open strings $(F 1)$ stretched between them should be the $F 1-\bar{F} 1$ system with $D$-strings $(D 1)$ joining them while that of the $D 5-\bar{D} 5$ system with fundamental strings suspended between them should likewise be the NS5 - NS5 system with $D$-strings connecting them. (Indeed, the $S$-dual of $(0 \mid F 1, D 5)$, namely the intersection, $(0 \mid D 1, N S 5)$ is actually known to exist 99.) The point on which we should be careful here is that, since the fundamental open strings $(F 1)$ stretched between $D_{p}$ and $\bar{D}_{p}(p=1,5)$ develop tachyonic modes and hence 
are unstable, their $S$-dual partners, $D$-strings $(D 1)$ joining $F 1$ and $\bar{F} 1$ or $N S 5$ and $N \overline{S 5}$ should be unstable D1's in IIB theory possessing again tachyonic modes. This is illustrated in Fig.1. Along the line of Sen's conjecture then, one can anticipate that at the true vev

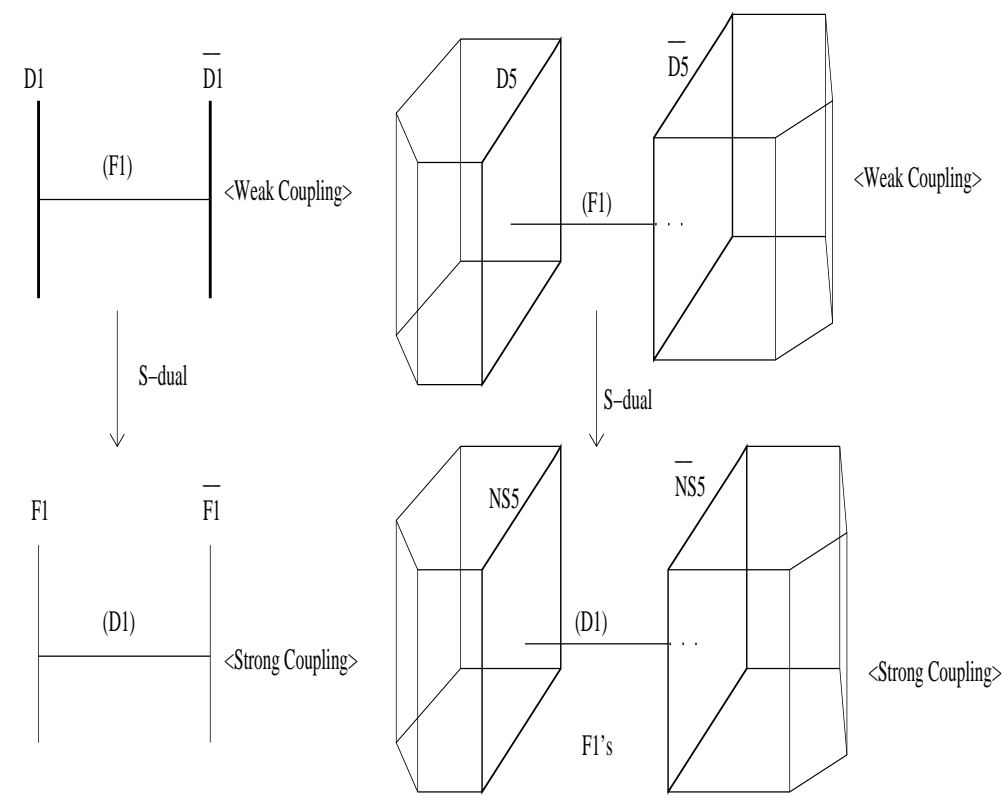

Figure 1: IIB theory $(F 1-\bar{F} 1, N S 5-\overline{N S 5})$ systems at strong coupling derived using $S$-duality.

$T=T_{0}$ of the tachyon on $D 1$ 's, the sum of the tensions of $N S$-branes and the (negative) minimum potential energy of the tachyon should exactly be zero, $V\left(T_{0}\right)+2 M_{N S}=0$, which is just the $S$-dual of the eq.(12) given earlier. Indeed, particularly the fact that the $D 1$ 's stretched between IIB $N S 5$ and $N \bar{S} 5$ possesses tachyonic mode and hence are responsible for the quantum instability of this system has been pointed out earlier in the literature invoking the self $S$-dual nature of IIB theory [5] just as discussed above or using the fact [4 that this IIB system is the T-dual of the IIA system of D2's stretched between IIA NS5 and NS5 whose quantum instability, in turn, is inherited from that of the M-theory system of $M 2$ 's stretched between $M 5$ and $\bar{M} 5[8]$. Thus a new ingredient in the present study is that this quantum description has been extended to the $F 1-\bar{F} 1$ pair as well in which $D 1$ 's stretched between them are again responsible, via their tachyonic modes, for the quantum instabilities.

\subsection{Stringy description of the instability at weak coupling}

The discussion so far, however, is valid only at strong coupling. Namely note that we have derived the stringy (or brany) description for the instabilities in the $N S N S$-charged 
brane-antibrane systems in this IIB theory using basically the $S$-duality by which the weak coupling picture of instabilities in $R R$-charged brane-antibrane systems have been mirrored to the strong coupling picture of those in NSNS-charged ones. As such, strictly speaking, this stringy (or brany) description for the instabilities in the NSNS-charged brane-antibrane systems holds true in the strong coupling regime. And since the systems are not BPS, the extrapolation of this particular description to the weak coupling regime would not be safe, and more careful treatment there is required. Thus in the following, we attempt at providing the origin of quantum instabilities of the same IIB systems at weak coupling. To this end, first notice that the tensions of fundamental string $(F 1)$ and $D$-string $(D 1)$ are given respectively by $T_{F 1}=1 /\left(2 \pi \alpha^{\prime}\right), T_{D 1}=1 /\left(2 \pi \alpha^{\prime} g_{s}\right)$ with $\alpha^{\prime}=l_{s}^{2}$ and $g_{s}$ being the string length squared and the string coupling respectively. At weak coupling $g_{s} \rightarrow 0$, which is the familiar case, the $D$-string gets heavy and the fundamental string is the lightest object in the theory and hence one should identify the $D$-string instability (as that of $D 1-\bar{D} 1$ system) as originating from the tachyonic modes of the fundamental strings ending on the $D$-string. And the weak coupling description of the unstable $D 1-\bar{D} 1$ system depicted in Fig. 1 given earlier exhibits such a situation. At strong coupling $g_{s} \rightarrow \infty$, however, now the fundamental string is heavy and the $D$-string becomes the lightest object in the theory. Thus the vibrating modes of the $D$-string can be seen in perturbation theory and this is why one can identify the $D$-string instability in terms of its own lowest-lying tachyonic mode as discussed above and in the literature [4, 5]. Now, precisely this last point provides the clue to our main question here as to the origin of quantum instabilities of type IIB $N S N S$-charged brane-antibrane systems at weak coupling. Namely as discussed above and shown in Fig.1 (the strong coupling side), at stong coupling, it is the tachyonic mode of the light $D$-string itself that is responsible for the quantum instability of the unstable, heavy fundamental string (such as that of $F 1-\bar{F} 1$ system). Now, consider the $(D 1-\bar{D} 1, D 5-\bar{D} 5)$ systems at strong coupling. Then the first thing that would come to one's mind is that their quantum instabilities might come from the $F$-strings stretched between them. For reasons just stated, however, this is not really the case as the $F$-string is now heavy and hence does not admit perturbative description for its vibrational modes. Instead, the instabilities essentially come from the tachyonic modes of light $D$-strings ending on this heavy $F$-string. And of course this would be true because now the $D$-string is the lightest object in the theory and it does have intersection with the $F$ string, i.e., $(0 \mid F 1, D 1)$. This realization of the role played by the light $D$-strings (ending on the heavy $F$-string generally stretched between $D_{p}$ and $\bar{D}_{p}$ ) as an actual source of quantum instabilities at strong coupling is what distinguishes the situation for the case at hand from 
what happens in the familiar weak coupling case discussed in the previous subsection and in the literature [4, 5]. Finally, then, the origin of instabilities in IIB theory $(F 1-\bar{F} 1$, $N S 5-N \bar{S} 5)$ systems at weak coupling can be deduced from that of $(D 1-\bar{D} 1, D 5-\bar{D} 5)$ systems at strong coupling just discussed again via $S$-duality. Namely under $S$-duality, this picture of unstable $(D 1-\bar{D} 1, D 5-\bar{D} 5)$ systems at strong coupling is mapped into the IIB theory $(F 1-\bar{F} 1, N S 5-N \bar{S} 5)$ systems at weak coupling in which heavy $D$-string stretched between $(F 1-\bar{F} 1, N S 5-N \bar{S} 5)$ has light $F$-strings ending on it whose lowest-lying tachyonic modes ultimately represent the quantum instabilities of these $N S N S$-chaged brane-antibrane systems. And clearly this is consistent with the familiar weak coupling description discussed earlier in which the tachyonic mode of the fundamental string is responsible for the quantum instability of the heavy $D$-string (and of other non-BPS $D_{p}$-branes as well). This suggestion for the origin of quantum instabilities of the NSNS-charged brane-antibrane systems at weak coupling has been depicted in Fig.2. Therefore for the $R R$-charged brane-antibrane

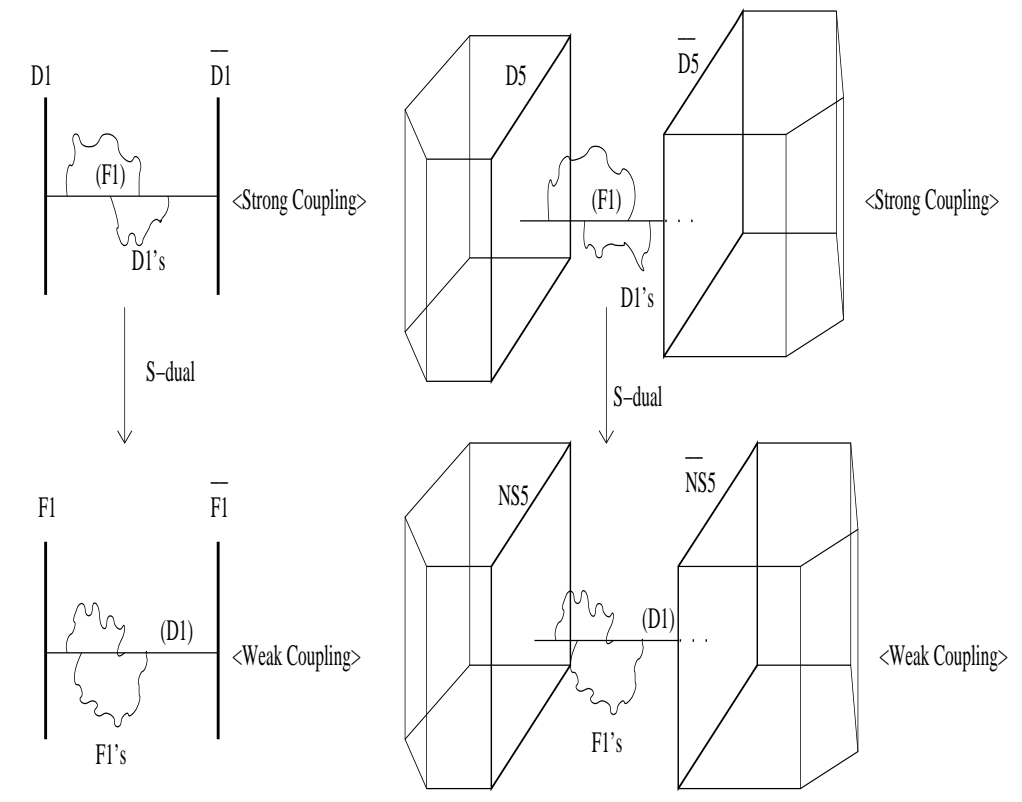

Figure 2: IIB theory $(F 1-\bar{F} 1, N S 5-N \bar{S} 5)$ systems at weak coupling derived using $S$-duality.

systems at strong coupling, one should expect

$$
\tilde{V}\left(\tilde{T}_{0}\right)+2 M_{D 1}+M_{F 1}=0, \quad \tilde{V}\left(\tilde{T}_{0}\right)+2 M_{D 5}+\frac{1}{\alpha^{\prime 2}} M_{F 1}=0
$$

for $D 1-\bar{D} 1, D 5-\bar{D} 5$ systems respectively and for their $S$-dual, NSNS-charged braneantibrane systems at weak coupling, one should expect

$$
\tilde{V}\left(\tilde{T}_{0}\right)+2 M_{F 1}+M_{D 1}=0, \quad \tilde{V}\left(\tilde{T}_{0}\right)+2 M_{N S 5}+\frac{1}{\alpha^{\prime 2}} M_{D 1}=0
$$


for $F 1-\bar{F} 1, N S 5-\overline{N S} 5$ systems respectively and where $M_{F 1}=T_{F 1}=1 / 2 \pi \alpha^{\prime}$ and $M_{N S 5}=$ $T_{N S 5}=1 /(2 \pi)^{5} \alpha^{\prime 3} g_{s}^{2}$. Note that the last term, the $F(D)$-string tension $M_{F 1}=T_{F 1}\left(M_{D 1}=\right.$ $\left.T_{D 1}\right)$ in eq.(13 (14)) (up to the factor $1 / \alpha^{\prime 2}$, being introduced for dimensional consideration), which was negligible at weak (strong) coupling, is now no longer so. $\tilde{V}\left(\tilde{T}_{0}\right)$ is the (negative) minimum of potential of the tachyon arising in the spectrum of open $D(F)$-strings ending on unstable $F(D)$-strings. Obviously, it is determined separately for $D 1-\bar{D} 1(F 1-\bar{F} 1)$ and $D 5-\bar{D} 5(N S 5-N \bar{S} 5)$ pairs and should be different in its structure and hence in value from $V\left(T_{0}\right)$ given in eq.(12) in the $R R$-charged brane-antibrane systems at weak coupling. And this is our proposal for the quantum, stringy description for the instabilities in the $N S N S$-charged $(F 1-\bar{F} 1, N S 5-N \bar{S} 5)$ systems at weak coupling in IIB theory that has remained unexplained thus far. Lastly, it is worthy of note that there is an important lesson to be learned from this study of instabilities in the IIB theory $R R$-charged braneantibrane systems at weak string coupling. It is the fact that the original Sen's conjecture for the $R R$-charged brane-antibrane annihilation via the tachyon condensation represented by $V\left(T_{0}\right)+2 M_{D}=0$ given above has its relevance only at weak coupling. And at strong

coupling, for which the fundamental open strings stretched between $D_{p}$ and $\bar{D}_{p}$ in IIA/IIB theories becomes relatively heavy and hence should have much lighter $D$-strings ending on them as an indicator for their quantum instability, the corresponding equation representing Sen's conjecture should be given instead by the ones in eq.(13) with the tachyon arising in the spectrum of open $D$-string instead. Thus in this way we extended Sen's conjecture at weak coupling to strong coupling as well.

\section{Instabilities in IIA theory $(F 1-\bar{F} 1, N S 5-N \bar{S} 5)$ sys- tems}

Since the $N S N S$-charged $F 1$ and $N S 5$ arise in the spectrum of both IIA and IIB theories, the analysis of instabilities would be incomplete unless we discuss the IIA theory case as well. Thus we now turn to the discussion of the instabilities in the $(F 1-\bar{F} 1, N S 5-N \bar{S} 5)$ systems in type IIA theory.

\subsection{Supergravity description of the instability}

For the present case of IIA theory, since the relevant string duality to employ is the M/IIA duality, in the following we demonstrate that starting with the unstable M-theory braneantibrane solutions, one can perform appropriate KK-reductions along the M-theory circle 
direction to obtain non-BPS $F 1-\bar{F} 1$ and $N S 5-\overline{N S} 5$ solutions. Now starting with the supergravity solution representing the system of $M 2-\bar{M} 2$ pair in $D=11$ [3],

$$
\begin{aligned}
d s_{11}^{2} & =H^{-2 / 3}\left[-d t^{2}+\sum_{i=1}^{2} d x_{i}^{2}\right]+H^{1 / 3}\left[\sum_{m=3}^{7} d x_{m}^{2}+\left(\Delta+a^{2} \sin ^{2} \theta\right)\left(\frac{d r^{2}}{\Delta}+d \theta^{2}\right)+\Delta \sin ^{2} \theta d \phi^{2}\right] \\
A_{[3]}^{11} & =-\left[\frac{2 m a \cos \theta}{\Sigma}\right] d t \wedge d x_{1} \wedge d x_{2}
\end{aligned}
$$

again with the "modified" harmonic function given by $H(r)=\Sigma /\left(\Delta+a^{2} \sin ^{2} \theta\right)$ and performing the KK-reduction along a direction longitudinal to the $M 2(\bar{M} 2)$ brane worldvolume, $x_{2}$,

$$
\begin{aligned}
d s_{11}^{2} & =e^{-\frac{2}{3} \phi} d s_{10}^{2}+e^{\frac{4}{3} \phi}\left(d x_{2}+A_{\mu} d x^{\mu}\right)^{2}, \\
A_{[3]}^{11} & =A_{[3]}^{I I A}+B_{[2]} \wedge d x_{2}
\end{aligned}
$$

one can obtain the supergravity solution representing the system of $F 1-\bar{F} 1$ pair given earlier in eq.(7) in the previous section. Next, starting with the $M 5-\bar{M} 5$ solution in $D=11$ [3],

$$
\begin{aligned}
d s_{11}^{2} & =H^{-1 / 3}\left[-d t^{2}+\sum_{i=1}^{5} d x_{i}^{2}\right]+H^{2 / 3}\left[d x_{6}^{2}+d x_{7}^{2}+\left(\Delta+a^{2} \sin ^{2} \theta\right)\left(\frac{d r^{2}}{\Delta}+d \theta^{2}\right)+\Delta \sin ^{2} \theta d \phi^{2}\right] \\
F_{[4]}^{11} & =\frac{2 m r a \sin ^{2} \theta\left(r^{2}+a^{2} \cos ^{2} \theta\right)}{\left(\Delta+a^{2} \sin ^{2} \theta\right)^{2}} d x_{6} \wedge d x_{7} \wedge d r \wedge d \phi \\
& -\frac{4 m r a \sin \theta \cos \theta \Delta}{\left(\Delta+a^{2} \sin ^{2} \theta\right)^{2}} d x_{6} \wedge d x_{7} \wedge d \theta \wedge d \phi
\end{aligned}
$$

where again $H(r)=\Sigma /\left(\Delta+a^{2} \sin ^{2} \theta\right)$ and carrying out the KK-reduction along a direction transverse to the $M 5(\bar{M} 5)$ brane worldvolume, $x_{7}$,

$$
\begin{aligned}
d s_{11}^{2} & =e^{-\frac{2}{3} \phi} d s_{10}^{2}+e^{\frac{4}{3} \phi}\left(d x_{7}+A_{\mu} d x^{\mu}\right)^{2}, \\
F_{[4]}^{11} & =F_{[4]}^{I I A}+H_{[3]}^{m} \wedge d x_{7}
\end{aligned}
$$

one can arrive at the $N S 5-\overline{N S} 5$ solution with $H_{[3]}^{m}=d B_{[2]}^{m}$ given earlier in eq.(9). Again, the fact that these M-theory brane-antibrane pair or the IIA theory $F 1-\bar{F} 1$ and $N S 5-N \bar{S} 5$ pairs represented by the supergravity solutions given above indeed exhibit semi-classical instabilities in terms of the appearance of the conical singularities can be found in detail in our earlier work [3].

\subsection{Stringy description of the instability at strong coupling}

We now move on to the quantum, stringy perspective. In order eventually to identify the quantum entity that is supposed to take over the semi-classical instability as the inter-brane 
distance gets smaller, say, toward the string scale $\sim \sqrt{\alpha^{\prime}}=l_{s}$, in the $(F 1-\bar{F} 1, N S 5-N \bar{S} 5)$ systems in type IIA theory, we start with their M-theory counterparts. Recall first that the $D=11$ M-theory possesses 5 -types of brane solutions ;

$$
M \text { - wave, } \quad M 2, \quad M 5, \quad M K K-\text { monopole, } \quad M 9
$$

and particularly the intersection rules known among $M 2$ and $M 5$ branes are given by [9] ;

$$
(0 \mid M 2, M 2), \quad(1 \mid M 2, M 5), \quad(1 \mid M 5, M 5), \quad(3 \mid M 5, M 5) .
$$

Thus from these intersection rules, we can deduce the following "triple" intersections. (Henceforth $M 5-(M 2)-\bar{M} 5$, for example, indicates the configuration in which (M2) is stretched between $M 5$ and $\bar{M} 5$.)

(I) $[M 2-(M 2)-\bar{M} 2] \rightarrow[F 1-(D 2)-\bar{F} 1]$

$$
\begin{array}{ll|llllllllll}
M 2: & 0 & 1 & 2 & - & - & - & - & - & - & - & - \\
\bar{M} 2: & 0 & 1 & 2 & - & - & - & - & - & - & - & - \\
(M 2): & 0 & - & - & 3 & 4 & - & - & - & - & - & -
\end{array}
$$

where we used $(0 \mid M 2, M 2)$ and $(0 \mid M 2, \bar{M} 2)$. Using now the M/IIA-duality, consider the KK-reduction following the compactification along the direction $x_{2}$ longitudinal both to $M 2$ and $\bar{M} 2$ but transverse to $(M 2)$ worldvolume to get

$$
\begin{array}{ll|lllllllll}
F 1: & 0 & 1 & - & - & - & - & - & - & - & - \\
\bar{F} 1: & 0 & 1 & - & - & - & - & - & - & - & - \\
(D 2): & 0 & - & 2 & 3 & - & - & - & - & - & -
\end{array}
$$

which is consistent with the known intersection rule $(0 \mid F 1, D 2)$.

(II) $[M 5-(M 2)-\bar{M} 5] \rightarrow\left[N S 5-(D 2)-N_{S} 5\right]$

$$
\begin{aligned}
& \begin{array}{ll|llllllllll}
M 5: & 0 & 1 & 2 & 3 & 4 & 5 & - & - & - & - & -
\end{array} \\
& \begin{array}{ll|lllllllllll}
\bar{M} 5 & : & 0 & 1 & 2 & 3 & 4 & 5 & - & - & - & - & -
\end{array}
\end{aligned}
$$

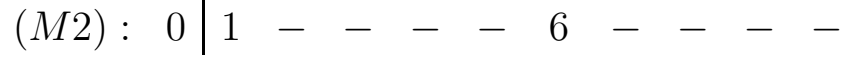

where we used $(1 \mid M 2, M 5)$ and $(1 \mid M 2, \bar{M} 5)$. In this time, we consider the compactification followed by the KK-reduction along the direction $x_{7}$ transverse both to $M 5(\bar{M} 5)$ and $(M 2)$ worldvolumes to arrive at

$$
\begin{array}{ll|lllllllll}
N S 5 & 0 & 1 & 2 & 3 & 4 & 5 & - & - & - & - \\
N S 5: & 0 & 1 & 2 & 3 & 4 & 5 & - & - & - & - \\
(D 2): & 0 & 1 & - & - & - & - & 6 & - & - & -
\end{array}
$$

which is consistent with the known intersection rule $(1 \mid D 2, N S 5)$ [9. Note here that for this case (II), one may instead consider the KK-reduction along the direction $x_{6}$ which is 
transverse to $M 5(\bar{M} 5)$ but longitudinal to the $(M 2)$ worldvolume to get $N S 5-(F 1)-N \bar{S} 5$ consistently with the known intersection rule $(1 \mid F 1, N S 5)$ 9]. This last option, however, is irrelevant to the present discussion of the quantum interpretation of the instability in $N S 5-\overline{N S} 5$ system since $F 1$ here is embedded in the $N S 5$ and $\overline{N S} 5$ entirely. Now since the M-theory membrane ((M2)) stretched between $M 2$ and $\bar{M} 2$ or $M 5$ and $\bar{M} 5$ should represent quantum instability presumably in terms of "string-like" tachyonic modes arising on it as suggested by Yi [8], their M/IIA-dual partners, i.e., the (D2)-branes connecting $F 1$ and $\bar{F} 1$ or NS5 and NS5 should be unstable D2's in IIA theory possessing again tachyonic modes. It is also worthy of note that for the case (I), the KK-reduction along the direction, say, $x_{3}$ transverse to $M 2(\bar{M} 2)$ but longitudinal to $(M 2)$ would yield $D 2-(F 1)-\bar{D} 2$, while for the case (II), the KK-reduction along the direction $x_{1}$ longitudinal both to $M 5(\bar{M} 5)$ and $(M 2)$ would lead to $D 4-(F 1)-\bar{D} 4$. Clearly, these are the familiar $R R$-charged brane-antibrane systems in type IIA theory in which the quantum, stringy description of the instabilties is given in terms of the open string tachyon condensation in the sense of Sen's conjecture. And it is precisely this point that provides the logical ground on which one can argue that the (M2)-brane stretched between $M 2$ and $\bar{M} 2$ or $M 5$ and $\bar{M} 5$ would develop "string-like" tachyonic modes (or tachyonic strings) and hence represent quantum instabilities in these M-theory brane-antibrane systems. Then again along the line of Sen's conjecture, one immediately anticipates $\bar{V}\left(\bar{T}_{0}\right)+2 M_{N S}=0$. As mentioned earlier when we discussed the type IIB-theory case, the argument particularly on the origin of instabilities in IIA theory $N S 5-N \bar{S} 5$ system precisely of this sort has been pointed out in the literature [4, 5]. Thus again a new ingredient in the present work is that this quantum description has been extended to the $F 1-\bar{F} 1$ pair as well (see Fig.3.).

\subsection{Stringy description of the instability at weak coupling}

The problem is, however, that the discussion thus far is again valid only at strong coupling and cannot be safely extrapolated to the weak coupling regime since the systems under consideration are not BPS. Namely note that we have derived the stringy (or brany) description for the instabilities in the $N S N S$-charged brane-antibrane systems in IIA theory using basically the the M/IIA-duality by which the instabilities in the M-theory braneantibrane systems have been dualized to the strong coupling picture of their 10-dimensional $N S N S$-charged counterparts. As before therefore, in the following we attempt to provide the origin of quantum instabilities of the IIA $N S N S$ brane-antibrane systems at weak string coupling. Unlike the strong coupling case discussed above, however, here we cannot employ 

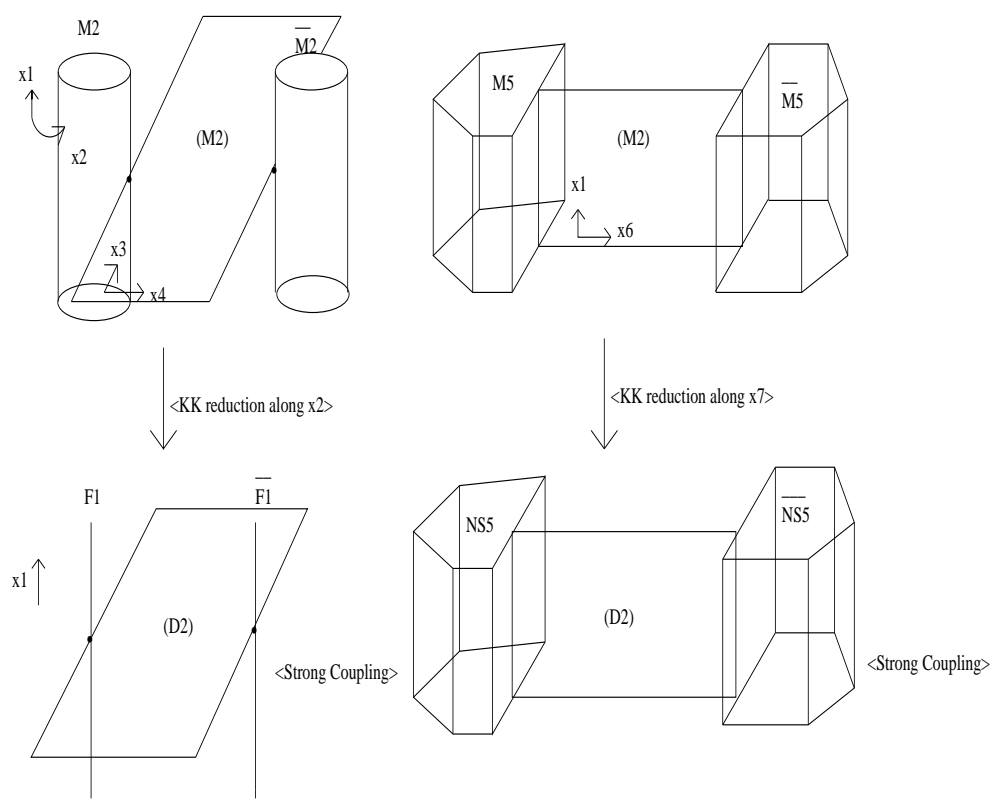

Figure 3: IIA theory $(F 1-\bar{F} 1, N S 5-\overline{N S 5})$ systems at strong coupling derived using M/IIA-duality.

the M/IIA-duality as there is no M-theory dual for weak coupling limit of type IIA string theory. Thus we should now consider some other way and it turns out that the origin of quantum instabilities of the IIA NSNS brane-antibrane systems at weak string coupling can be unveiled by taking the T-dual of its IIB theory counterpart discussed in sect.2.3. Namely, start with our earlier study of the origin of quantum instabilities of IIB NSNS brane-antibrane systems at weak coupling. First, for the IIB $F 1-\bar{F} 1$ case, in which the $D$-string stretched between $F 1$ and $\bar{F} 1$ is heavy and hence the tachyonic modes of the light $F$-strings ending on it are responsible for quantum instabilities, we take a $T$-dual of this system along a direction, say $x_{3}$, which is transverse to all $F 1$ 's, $\bar{F} 1$ and $D 1$. Then one ends up with the system in which now heavy $D 2$ stretched between $F 1$ and $\bar{F} 1$ has light $F$-strings ending on it whose lowest-lying tachyonic modes represent the quantum instability of this system. And this is because under this particular $T$-dual, all $F 1$ 's and $\bar{F} 1$ are mapped to themselves. Next, for the IIB $N S 5-N S 5$ case, take a $T$-dual along a direction, say $x_{1}$, which is transverse to all $F 1$ 's and $D 1$ but longitudinal to $N S 5$ and $N \bar{S} 5$. In this way, one is left with the system in which heavy $D 2$ stretched between $N S 5$ and $N S 5$ has light $F$-strings ending on it whose lowest-lying tachyonic modes again represent the quantum instability of the system. And this is because under this particular T-dual, all F1's and NS5 and NS5 are mapped to themselves. This suggestion has been depicted in Fig.4. Therefore at weak 

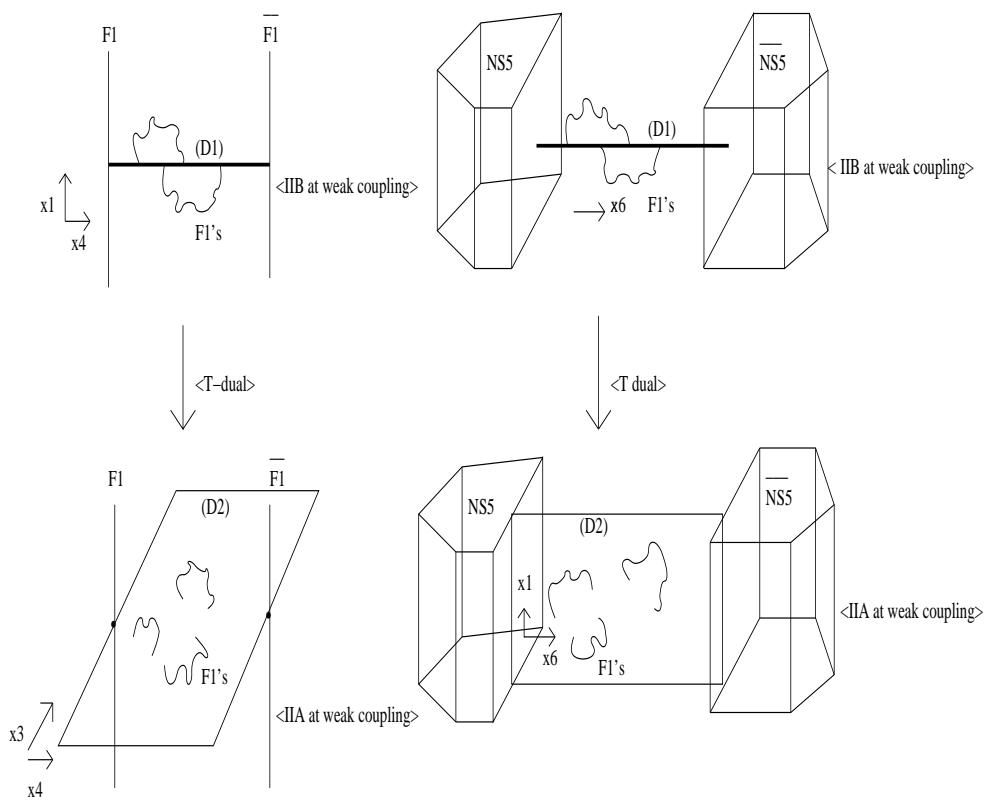

Figure 4: $\quad$ IIA theory $(F 1-\bar{F} 1, N S 5-N \bar{S} 5)$ systems at weak coupling derived using T-duality.

coupling, one should expect instead

$$
\bar{V}\left(\bar{T}_{0}\right)+2 M_{F 1}+\alpha^{\prime 1 / 2} M_{D 2}=0, \quad \bar{V}\left(\bar{T}_{0}\right)+2 M_{N S 5}+\frac{1}{\alpha^{\prime 3 / 2}} M_{D 2}=0
$$

which are the $T$-dual versions of eq.(14). Note that the last terms, the D2-brane tension $M_{D 2}=T_{D 2}=1 /(2 \pi)^{2} \alpha^{\prime 3 / 2} g_{s}$, (again up to the $\alpha^{\prime}$-dependent factors being introduced for dimensional reason) which was negligible at strong coupling, is now no longer so. $\bar{V}\left(\bar{T}_{0}\right)$ is the (negative) minimum of potential of the tachyon arising in the spectrum of open fundamental strings ending on unstable D2-branes. Apparently, it is determined separately for $F 1-\bar{F} 1$ and $N S 5-\overline{N S} 5$ pairs and should be different from its IIB theory counterpart $\tilde{V}\left(\tilde{T}_{0}\right)$ given earlier in eq.(14) or from $V\left(T_{0}\right)$ appearing in the $R R$-charged brane-antibrane systems given in eq.(12). Before we leave this subsection, we comment on yet another possibility. That is, starting with the M-brane intersection rule $(1 \mid M 2, M-$ wave $)$ [9], one can, via the KKreduction along the direction longitudinal both to $M-$ wave and $M 2$, the intersection $(0 \mid F 1, D 0)$, which, in turn, implies the $F 1-(D 0)-\bar{F} 1$ situation. Thus at strong coupling, it would be the tachyonic mode of the light $D 0$ itself while at weak coupling, it would be that of the light $F$-strings ending on the heavy $D 0$, that could also be responsible for the quantum instability of IIA theory $F 1-\bar{F} 1$ system in addition to the situation associated with the $D 2$ discussed above. Analogous situation associated with IIA theory $N S 5-\overline{N S} 5$ system, namely the configuration $N S 5-(D 0)-N \bar{S} 5$, however, does not arise. This is because 
the potentially relevant intersection rule $(1 \mid M 5, M$ - wave) 9], leads, via the KK-reduction along the direction transverse both to $M-$ wave and $M 5$, to the intersection $(1 \mid N S 5, W)$, (with $W$ denoting the IIA theory $p p$-wave) which implies the $N S 5-(W)-N \overline{S 5}$ situation. Evidently, this configuration is not relevant for the present discussion as the IIA theory $p p$-wave, which is entirely embedded in NS5 (and NS5) is not known to be tachyonic. And this is our proposal for the quantum, stringy description for the instabilities in the NSNScharged $(F 1-\bar{F} 1, N S 5-N \bar{S} 5)$ systems at weak coupling in IIA theory that has not been discussed thus far.

\section{Concluding remarks}

Firstly, it is worthy of note that there is an important lesson we learned in the present study of quantum instabilities of $R R$ and $N S N S$-charged brane-antibrane systems. That is, at strong coupling, the fundamental open strings stretched between $R R$-charged $D_{p}$ and $\bar{D}_{p}$ in IIA/IIB theories become relatively heavy and hence should have much lighter $D$ strings ending on them as an indicator for their quantum instability. And via the $S$-dual of this picture, we then realised that at weak coupling, the $D$-strings stretched between $N S N S$-charged $F 1-\bar{F} 1$ and $N S 5-N \bar{S} 5$ systems in IIA/IIB theories become relatively heavy and hence should likewise have much lighter fundamental strings ending on them again as an indicator for their quantum instability. In this realisation, however, one might wonder if these $F 1^{\prime} s$ and $D 1^{\prime} s$ might form bound states and then add further instability to the system. In some intermediate coupling regime, there indeed may be possibility of forming such $F 1-D 1$ bound states that would spoil the validity of our suggestion stated above. To see this in some more detail, we follow the argument of Witten [10]. Consider the system of, say, a $F$-string and a $D$-string in parallel. The total tension of this system reads $T_{F 1}+T_{D 1}=\left(g_{s}+1\right) /\left(2 \pi \alpha^{\prime} g_{s}\right)$ which is certainly greater that that of a $F 1-D 1$ BPS bound state [10], $T_{F 1-D 1}=\sqrt{g_{s}^{2}+1} /\left(2 \pi \alpha^{\prime} g_{s}\right)$ for some finite, intermediate value of the coupling $g_{s}$. Thus this parallel configuration is not supersymmetric but it can lower its energy till it reaches the the BPS bound above and become eventually supersymmetric. Namely, the $F$-string breaks, its endpoints being attached to the $D$-string (or more generally one ends on the other) and then moves off to infinity. Meanwhile, $F$-string endpoints are charged under the $D$-string gauge field. Therefore, if this happens, a flux runs between the endpoints and hence the final configuration is a $D$-string with a parallel flux or in effect, a $D$-string with the $F$-string dissolved on it. And a detailed calculation [10] shows that the final tension then 
saturates the BPS bound. All this argument on the possibility of forming a $F 1-D 1$ bound state, however, is relevant at some intermediate coupling. At either very strong or very weak coupling regime, $F 1^{\prime} s$ and $D 1^{\prime} s$ have nearly negligible chance to form such bound states and hence this kind of situation becomes irrelevant to consider. To see this explicitly, consider again the system of $F 1$ and $D 1$ (i.e., one ending on the other) with the total tension being given by $T_{F 1}+T_{D 1}=1 /\left(2 \pi \alpha^{\prime}\right)+1 /\left(2 \pi \alpha^{\prime} g_{s}\right)$. At very strong coupling, it reduces just to that of $F 1$, i.e., $T_{F 1}=1 /\left(2 \pi \alpha^{\prime}\right)$ whereas at very weak coupling it becomes almost that of $D 1$, i.e., $T_{D 1}=1 /\left(2 \pi \alpha^{\prime} g_{s}\right)$. This means that either at very strong or at very weak coupling, the two cannot behave as a bound state but instead one is very light and provides a vibrational spectrum, particularly the tachyonic mode, while ending on the other heavy string. And certainly, we do not call this a bound state of two strings of comparable masses (tensions, for the case at hand).

Next, it seems relevant to check if the quantum description for the instabilities in the NSNScharged brane-antibrane systems in IIA/IIB theories discussed in the present work is indeed consistent with some known wisdom as of now. Thus we particularly focus on the case of $N S 5-N \bar{S} 5$ systems in IIA/IIB theories. The nature of $N S 5$-brane worldvolume dynamics uncovered thus far [1] can be summarized as follows. Using the worldvolume dynamics of $R R$-charged $D$-branes and invoking the string dualities, it has been derived that the nonchiral type IIA string theory gives rise to a chiral NS5-brane worldvolume theory with $(2,0)$ supersymmetry in 6-dimensions while the chiral type IIB string theory yields a non-chiral NS5-brane with $(1,1)$ supersymmetry. Thus the light fields on a single IIA NS5-brane worldvolume correspond to a tensor multiplet of 6-dimensional (2,0) SUSY, consisting of a self-dual $B_{\mu \nu}$ field and 5-scalars (and fermions). Meanwhile on a single IIB NS5-brane worldvolume, there is a vector multiplet consisting of a 6-dimensional gauge field and 4scalars (and fermions). The four of the 5-scalars in the tensor multiplet on the IIA NS5brane and the 4-scalars in the vector multiplet on the IIB NS5-brane describe fluctuations of the NS5-brane in the transverse directions. Next, the low energy worldvolume dynamics on a stack of $N$-coincident IIB $N S 5$-branes is a 6 -dimensional $(1,1) S U(N)$ SYM theory arising from the ground states of $D$-strings stretched between them. And the low energy worldvolume theory on a pile of $N$-coincident IIA $N S 5$-branes is a non-trivial field theory with $(2,0)$ SUSY in 6-dimensions. Particularly, it contains string-like low energy excitations corresponding to D2-branes stretched between them. Certainly this last point, namely that $D 1$-branes and D2-branes provide the low energy worldvolume dynamics on the NS5-branes in type IIB and IIA theories respectively, is indeed consistent with our findings in the present 
work. That is, unstable $D 1$ and unstable $D 2$ each with (at weak coupling) or without (at strong coupling) fundamental strings ending on each of them possessing tachyonic modes, are responsible for the quantum instabilities in the $N S 5-N \bar{S} 5$ pairs in type IIB and IIA theories respectively. Next, to repeat, the main purpose of the present work was to uncover concrete quantum interpretation of the instabilities in the NSNS-charged brane-antibrane systems in type IIA/IIB theories in terms of stringy description. And we were particularly interested in the case when the endpoint of the brane-antibrane annihilation is the string vacuum in which the supersymmetry is fully restored. Now, going back to the instabilities in the $R R$-charged brane-antibrane systems, there is, again according to Sen's conjecture [2], the other channel for the decay of $D_{p}-\bar{D}_{p}$ systems in which the supersymmetry is partially restored and there the endpoint is a stable $D_{(p-2)}$-brane realised as a topological soliton. To be a little more specific, starting with a non-BPS $D_{p}-\bar{D}_{p}$ system in type IIA/IIB theories, in the next step a non-BPS $D_{(p-1)}$-brane may result as the tachyonic kink on the brane-antibrane pair and then in the last stage a BPS $D_{(p-2)}$-brane can emerge again as the tachyonic kink on the non-BPS $D_{(p-1)}$-brane worldvolume. In this spirit, the NSNScharged NS5 - NS5 system in IIB theory may settle down to a BPS D3-brane since in this self $S$-dual IIB theory, $N S 5-N \bar{S} 5$ system is $S$-dual to $D 5-\bar{D} 5$ system and $D 3$ is $S$-dual to itself, as has been pointed out first by Yi $[8$. The decay of the $N S 5-N S 5$ system possibly to another lower-dimensional brane in IIA theory, on the other hand, should be treated in a rather different fashion as the $N S 5-N S 5$ pair in IIA theory does not have any link to $R R$-charged $D-\bar{D}$ pair. Here, the relevant string duality one can turn to is the M/IIA-duality. Thus from this point on, Yi [ $[$ ] also argued that the NS5 - NS5 pair in IIA theory may relax to a $D 2$-brane as it can be deduced from the $S^{1}$ compactification of a M-theory picture in which $M 5$ and $\bar{M} 5$ annihilate into a $M 2$. One can quantify this argument as follows. Consider the Chern-Simons term in the effective worldvolume theory action for $N S 5-N \bar{S} 5$ system which would essentially be the same as that for $M 5-\bar{M} 5$ system given by

$$
\int_{R^{5+1}} C_{[3]} \wedge H_{[3]}
$$

where $C_{[3]}$ and $H_{[3]}$ are the descendants (to $D=10$ ) of 3-form tensor potential of $D=11$ supergravity and the field strength of the 2-form living in the worldvolume of $M 5$-brane [8, 4] respectively. Now integrating over a localized and quantized magnetic flux $H_{[3]}$ on a transverse $R^{3}$, one ends up with

$$
\int_{R^{2+1}} C_{[3]}
$$


which is just the way how $C_{[3]}$ would couple to a $D 2$-brane implying that the localized and quantized magnetic $H$ flux should be identified with a $D 2$-brane. Note that this mechanism by which one can identify the $D 2$-brane as a topological soliton emerging from the tachyon condensation via the (non) abelian Higgs mechanism works both at strong and at weak couplings. But what distinguishes between the two coupling regimes is the fact that at strong coupling, the $D 2$-brane instability originates from tachyonic mode of the vibrational spectrum of $D 2$ itself which is indeed light whereas at weak coupling, it comes from tachyonic mode of the light $F 1$ 's ending on heavy $D 2$. This is a new finding made in the present work. In the present work, again we remind the reader that we were particularly interested in the case when the endpoint of both $R R$ and $N S N S$-charged brane-antibrane annihilations is the string vacuum in which the supersymmetry is fully restored. As pointed out by Sen [2], however, for the case of unstable $R R$-charged $D$-branes, there is other decay channel in which the final state is a stable BPS lower-dimensional $D$-brane with partially restored supersymmetry instead. More precisely, as we already mentioned above, a non-BPS $D_{p}-\bar{D}_{p}$ system in type IIA/IIB theories may decay and settle down to a BPS $D_{(p-2)}$-brane which is a topological soliton emerging from the tachyon condensation via the Higgs-type mechanism [8]. Now that we seem to be left with two different decay channels, the first leading to the string vacuum with fully restored supersymmetry while the second yielding a stable BPS lower-dimensional brane with partially restored supersymmetry. Obviously, these two decay channel are distinct since the endpoint of the second channel, namely a stable BPS lower-dimensional brane would never decay further to the vacuum spontaneously. One, then, would be puzzled and led to ask a question such as what would be the eventual fate of an unstable brane, or put differently, which channel would be favored (or more probable) over the other? And the same question may be asked for the case of NSNS-charged brane-antibrane decays such as the $N S 5-N \bar{S} 5$ system in IIA/IIB theories which can either annihilate to the string vaccum or decay to a $D 2$-brane or to a $D 3$-brane respectively. The relevant answer to this question seems to be that it would depend on which tachyon condensation mechanism happens to operate, namely between the one leading to the string vacuum and the other yielding the topological soliton via the Higgs-type mechanism. And for now one never knows which mechanism has better chance to work.

Lastly, we stress again that either for the annihilations of the NSNS-charged brane-antibranes in IIA/IIB theories or for the decays to lower-dimensional $D$-branes conjectured in the literature, their physical understanding can never be complete unless the concrete stringy description of the quantum instability both at strong and at weak couplings is given. And 
we believe that in the present work, we have provided such a comprehensive stringy interpretation.

\section{Acknowledgments}

This work was financially supported by the BK21 Project of the Korean Government. And the author would like to thank Sunggeun Lee for assistance to generate the figures.

\section{References}

[1] O. Bergman and M. R. Gaberdiel, Phys. Lett. B441 (1998) 133 ; A. Sen, JHEP 9809 (1998) 023 ; JHEP 9810 (1998) 021 ; JHEP 9812 (1998) 021 ; E. Witten, JHEP 9812 (1998) 019 ; P. Horava, Adv. Theor. Math. Phys. 2 (1999) 1373.

[2] A. Sen, JHEP 9808 (1998) 010 ; JHEP 9808 (1998) 012 ; JHEP 9912 (1999) 027 ; hep-th/9904207

[3] H. Kim, Nucl. Phys. B651 (2003) 143, hep-th/0208068 ; JHEP 0301 (2003) 080, hep-th/0204191

[4] L. Houart and Y. Lozano, Nucl. Phys. B575 (2000) 195 ; JHEP 0003 (2000) 031.

[5] K. Intriligator, M. Kleban and J. Kumar, JHEP 0102 (2001) 023.

[6] D. Youm, Nucl. Phys. B573 (2000) 223.

[7] W. B. Bonnor, Z. Phys. 190 (1999) 444.

[8] P. Yi, Nucl. Phys. B550 (1999) 214.

[9] E. Bergshoeff et al., Nucl. Phys. B494 (1997) 119 ; Class. Quant. Grav. 14 (1997) 2757 ; E. Bergshoeff, J. Gomis and P. K. Townsend, Phys. Lett. B421 (1998) 109.

[10] E. Witten, Nucl. Phys. B460 (1996) 335 ; see also, J. Polchinski, TASI Lectures on D-branes, hep-th/9611050.

[11] E. Witten, hep-th/9507121; A. Strominger, Phys. Lett. B383 (1996) 44 ; N. Seiberg, Nucl. Phys. Proc. Suppl. 67 (1998) 158 ; A. Giveon and D. Kutasov, Rev. Mod. Phys. 71 (1999) 983. 